\title{
Interlaminar stresses in corrugated laminates
}

\author{
C. Thurnherr ${ }^{\mathrm{a}, *}$, L. Ruppen ${ }^{\mathrm{a}}$, G. Kress ${ }^{\mathrm{a}}$, P. Ermanni ${ }^{\mathrm{a}}$ \\ ${ }^{a}$ Laboratory of Composite Materials and Adaptive Structures, Department of Mechanical \\ and Process Engineering, ETH Zürich, Tannenstr. 3, CH-8092 Zürich, Switzerland
}

\section{Abstract}

In axially loaded corrugated laminates consisting of more than one layer interlaminar stresses occur due to the curvature which potentially cause delamination of the laminate. Therefore, it is crucial to know how geometry and lay-up influence the interlaminar stress and hence the risk of delamination. In this paper, a parameter study is presented that studies the influence of corrugation amplitude and different lay-ups on interlaminar stresses. We considered two geometries, one consists of circular sections and the other is a sinusoidal shape. From the parameter study we can derive favorable configurations to minimize normalized interlaminar stresses. A numerical model is used to calculate the stress distribution in the cross-section of the corrugation. It considers a generalized plane strain state and uses a unit-cell approach. The model is validated with experiments in a tensile test using digital image correlation (DIC).

Keywords: Corrugated laminates, interlaminar stresses, anisotropic materials, flexible skins

\footnotetext{
${ }^{*}$ Corresponding author

Email address: thclaudi@ethz.ch (C. Thurnherr)
} 


\section{Introduction}

The geometry effects of singly-curved corrugated sheets create extreme anisotropy. As other authors [1,2] have suggested, corrugated sheets are ideal candidates for the design of flexible skins as a structural element in morphingwing design, where high stretch ability along the chord direction must be combined with high contributions to structural stiffness and strength along

the span direction of an airplane wing [3]. Other authors [4, 5] investigated the potential of morphing wing concepts to achieve higher control and flight performance at lower weight than conventional solutions. To achieve higher mass-specific stiffness and strength and higher limit strain, the base sheet should be a laminate made from carbon-fiber reinforced plastic (CFRP). A recent review article [6] summarizes the state-of-the-art of the research investigating corrugated sheet concepts emphasizing the relevance of these structures.

Flexible skins must not impair the aerodynamic properties of the morphing wings. It has been found that the aerodynamic behavior improves with decreasing corrugation amplitudes and periodic cell lengths [5, 7]. Smaller periodic lengths lead to higher laminate thickness-to-curvature ratios, which increase the local interlaminar stresses caused by the corrugation geometry. The interlaminar stresses can cause layer debonding at relatively low external loads [8]. The present work investigates the influence of corrugation parameters on the ratio of out-of-plane to in-plane stress components in order to identify, in section 4.3, design limits with respect to interlaminar strength. We focus on periodic corrugated structures, however, the considerations are in general also applicable for curved laminates. 
The analysis of corrugated structures made from anisotropic material can lead to enormous computational costs. Conventional finite element methods are not efficient if the structure contains many periods of the corrugations and consists of many layers. Dayyani et al. presented a detailed nonlinear finite element study of corrugated structures [9], but this would be too time consuming for our purposes since we aim to perform parameter studies. Therefore, fast models are needed to calculate the mechanical response. Several analytical models exist to analyze the initial stiffness corrugated structures. Xia et al. suggested a homogenization model to calculate the stiffness matrix for thin balanced laminates, thus ignoring the coupling stiffness matrix B [10,11]. Winkler et al. [12] proposed an equivalent model for circular sections. Mohammadi et al. suggested an analytical model for trapezoidal shapes based on a homogenization approach [13]. These models are only valid for thin laminates and therefore are note appropriate for the present study where we investigate relatively thick laminates. Hence, a numerical approach is more suitable for the present work. Peng et al. [14] introduced a mesh-free Galerkin model to calculate the elastic stiffness behavior of trapezoidal and sinusoidal shaped corrugations. The model was extended by Liew et al. [15] to simulate the nonlinear response of corrugated laminates.

Various models exist to calculate the stress distribution in curved structures. An analytical model to predict the stress in curved laminates was suggested by Roos et al. and Kress et al. respectively [16, 17]. Fraternali et al. proposed a one-dimensional finite element model to calculate interlaminar stresses in curved beams that are loaded in three-point bending [18]. Shenoi et al. presented an analytical model based on elasticity-theory to 
predict through-thickness stresses in curved laminates and sandwich panels [19]. Gonzalez-Cantero et al. introduced a semi-analytic method to predict interlaminar stresses in curved beams with constant curvature [20]. All these models are not specifically developed to analyze periodic corrugated structures, are not suitable for arbitrary shapes, do only consider certain load cases or are restricted to single components of the interlaminar stress.

Recently, Kress et al. suggested a numerically efficient model using a unit-cell approach and a generalized plane strain state to model corrugations of any shape, thickness and with arbitrary lay-ups [21, 22, 23]. The model is suitable for all load cases describing the full mechanical response of the structure. This model is applied and extended in this paper in order to analyze the spatial stress distribution in corrugated laminate cross-sections.

The objectives of this paper are to investigate the influence of geometry and laminate lay-up onto interlaminar stresses. We further ask the question whether we can identify favorable geometries in order to minimize the interlaminar stresses. We compare a sinusoidal shaped corrugation to a corrugation consisting of circular sections. We consider four different lay-ups consisting of pure twill or UD layers and combinations of them. A parameter study is presented where we investigate the influence of the corrugation amplitude and the lay-up on the mechanical behavior, namely the interlaminar stresses and the axial stiffness, for the different geometries. Further, we present in this paper an experimental validation of the used numerical model where we measured displacements and strains.

The following section of the paper introduces the numerical model, geometry definition and load cases. Then the experiments are described and the 
experimental results are presented and discussed. The next section describes the parameter study and shows and discusses the numerical results of the simulations, including the stress distribution for certain examples and the results of the parameter study. The paper closes with a conclusion of the present work.

\section{Numerical model}

We used a numerical model that is able to calculate the mechanical response of corrugated laminates with arbitrary thickness and lay-up. Our model is based on the FEM model that was suggested by Kress and Winkler [21, 22, 23]. The model uses a unit-cell approach and a generalized plane strain state to reduce the computational costs. The generalized plane strain state assumes that the stresses and strains do not change with respect to the out-of-plane $x$ direction. It is derived from a simplified mechanical equilibrium:

$$
\begin{aligned}
& \tau_{y x, y}+\tau_{z x, z}=0 \\
& \sigma_{y, y}+\tau_{z y, z}=0 \\
& \tau_{y z, y}+\sigma_{z, z}=0
\end{aligned}
$$

Uniform strains $\hat{\epsilon}_{x}^{0}$, uniform bending about $y \hat{\epsilon}_{x}^{1}$ and twist $\hat{\epsilon}_{x y}^{1}$ are used to force deformations. They are compatible with the generalized-plane-strain assumption and they lead to a displacement field of the form: 


$$
\begin{aligned}
& u_{x}=u_{x}(y, z)+x\left(\hat{\epsilon}_{x}^{0}+z \hat{\epsilon}_{x}^{1}\right)+\frac{1}{2} y z \hat{\epsilon}_{x y}^{1} \\
& u_{y}=u_{y}(y, z)+\frac{1}{2} z x \hat{\epsilon}_{x y}^{1} \\
& u_{z}=u_{z}(y, z)-\frac{1}{2} x^{2} \hat{\epsilon}_{x}^{1}-\frac{1}{2} x y \hat{\epsilon}_{x y}^{1}
\end{aligned}
$$

This displacement solution consists of an inner FE solution $u_{i}(y, z)$ and the prescribed strains $\hat{\epsilon}_{i}^{0,1}$. The inner FE solution is not a function of the transverse direction $x$. Hence, it is sufficient to calculate the FE solution at one cross-section position only. This reduces the computational costs.

The strains are calculated from the displacement solution using the linearized kinematic relations,

$$
\boldsymbol{\epsilon}=\boldsymbol{L u}+\hat{\boldsymbol{\epsilon}}
$$

where the linear differential operator $\boldsymbol{L}$

$$
\boldsymbol{L}^{T}=\left[\begin{array}{cccccc}
0 & 0 & 0 & \frac{\partial}{\partial y} & 0 & 0 \\
0 & \frac{\partial}{\partial y} & 0 & \frac{\partial}{\partial z} & 0 & 0 \\
0 & 0 & \frac{\partial}{\partial z} & 0 & \frac{\partial}{\partial z} & \frac{\partial}{\partial y}
\end{array}\right]
$$

is adapted to the plane-strain assumption as it contains no derivatives with respect to $x$ and $\hat{\boldsymbol{\epsilon}}$ contains the prescribed macro strains:

$$
\hat{\boldsymbol{\epsilon}}^{T}=\left[\hat{\epsilon}_{x}^{0}+\hat{\kappa}_{x} z 00000 \hat{\kappa}_{x y} z\right]
$$

The stresses are calculated from the strains using the generalized Hook's law:

$$
\boldsymbol{\sigma}_{x y z}=\boldsymbol{C \epsilon}
$$


where $\boldsymbol{\sigma}_{x y z}$ and $\boldsymbol{\epsilon}$ denote the stress and strain vector, respectively. $\boldsymbol{C}$ represent the material law for orthotropic materials in global coordinates.

The above expressions for strains and stresses are used to formulate the weak variational form

$$
\int_{\Omega} \delta \boldsymbol{u}^{T} \boldsymbol{L}^{T} \boldsymbol{C}(\boldsymbol{L} \boldsymbol{u}+\hat{\boldsymbol{\epsilon}}) d \Omega-\int_{\Gamma} \delta \boldsymbol{u}^{T} \boldsymbol{\Phi} \hat{\boldsymbol{\sigma}} d \Gamma=0
$$

which is transformed by the finite-element method to a numerical system of equations where the unknown mesh-node displacements $\tilde{\boldsymbol{u}}$ respond to the forces contained in the right-hand-side $\boldsymbol{r}$ and the structural properties are mapped with the stiffness matrix $\boldsymbol{K}$ :

$$
\boldsymbol{K} \tilde{\boldsymbol{u}}=\boldsymbol{r} .
$$

The standard notation of the stiffness matrix $\mathrm{K}$ is

$$
\boldsymbol{K}=\sum_{k=1}^{N_{e l}} \int_{\Omega_{e l}} \boldsymbol{B}^{T} \boldsymbol{C} \boldsymbol{B} d \Omega
$$

where $N_{e l}$ is the number of finite elements in the mesh, $\Omega_{e l}$ is element domain, $\boldsymbol{B}$ relates node displacements to element strain distributions,

$$
\epsilon=B \tilde{u}=L \Phi \tilde{u}
$$

where $\boldsymbol{\Phi}$ are the element displacement approximation functions. The righthand side reflects natural boundary conditions and the macro strains to be described for the generalized plane-strain condition,

$$
r=\sum_{k=1}^{N_{e l}} \int_{\Gamma} \boldsymbol{\Phi}^{T} \hat{\boldsymbol{\sigma}} d \Gamma-\sum_{k=1}^{N_{e l}} \int_{\Omega_{e l}} \boldsymbol{B}^{T} \boldsymbol{C} \hat{\boldsymbol{\epsilon}} d \Omega
$$

where $\hat{\boldsymbol{\sigma}}$ contains the tractions specified on the boundary $\Gamma$. 
The form allows for a planar mesh where each node carries three displacement degrees-of-freedom. By using a unit-cell, we assume that the corrugation pattern is periodic and we apply periodic boundary conditions on both ends.

To evaluate the stresses and strains at an optimal location in the finite element, the Barlow points are well known and used as a standard procedure [24]. In our case the Barlow point coincide with the three times three Gauss points. Using the constitutive law, we can calculate the stresses from the strains. We define the stresses in the corrugated laminate according to figure 1 where components with index $s$ correspond to intralaminar and with index $t$ to interlaminar stresses.

Figure 1: Stress definition in local coordinate system $s, t$

In the present work we validate the model with experiments. We investigate both the displacement and strains. Further, we check the fulfillment of the mechanical equilibrium conditions like stress-free surfaces to verify the stress solution.

\subsection{Geometry}

We investigate two cross-section geometries, one consists of circular sections and the other is a sinusoidal shape as shown in figure 2. To describe the geometry of the corrugation we use the periodic cell-length $p$, the amplitude $h$ and the laminate thickness $t$. For the circular sections the radius $R$ can be calculated using $R=\frac{16(h / p)^{2}+1}{32 h / p}[21]$. 
Figure 2: Corrugation geometry: a) circular sections b) sinusoidal shape [25]

We use a global and a local coordinate system. In the global coordinate system, the $x$-axis points in direction transverse to the corrugation, the $y$-axis is in direction of the corrugation pattern and the $z$-axis points in direction of the amplitude as shown in figure 2. In the local coordinate system we have a coordinate $s$ pointing in direction along the mid-face shape and a coordinate $t$ perpendicular to $s$ in through-thickness direction as shown in figure 1 .

\subsection{Load case}

We consider a tensile load case where we force a displacement in $y$ direction that results in a total global strain of $1 \%$.

\section{Experimental model validation}

This section presents the conducted experiments that are necessary to validate the numerical model. First, the sample geometry and lay-up is presented, followed by the experimental set-up and measuring techniques. The last subsection shows the results and the discussion respectively.

\subsection{Sample Design}

The geometry of the sample was of circular shape with a periodic cell length $p$ of $60 \mathrm{~mm}$, an amplitude $h$ of $10 \mathrm{~mm}$ and a width of $280 \mathrm{~mm}$. The samples consisted of seven unit cells and eight layers as shown in figure 3 . 
Figure 3: Sample geometry

Figure 4 shows the three different lay-ups made from twill and UD material. We fabricated five samples with lay-up 1 and three with lay-ups 2 and 3 .

Figure 4: Three tested lay-ups made from twill and UD layers

We measured the thickness of the samples at four different points in each unit cell. We found an average thickness of 1.9 to $2 \mathrm{~mm}$ and a range of values between 1.8 and $2.1 \mathrm{~mm}$. These measurements might include excess resin.

As a material we used Twill Sigratex Prepreg CE 8201-200-45S and UD Sigratex Prepreg CE 1250-230-39 both from SGL Group. The lay-up angle was 90 degrees with respect to the $\mathrm{x}$-axis which means that the UD fibers pointed in s-direction along the mid-face-shape.

The Young's modulus in fiber direction does highly effect the simulation results. Therefore, it was measured according to the standard DIN EN ISO 527-5. For the Twill prepreg we found a mean value of $62.30 \mathrm{GPa}$ with a standard deviation of $2.74 \mathrm{GPa}$ and for the UD prepreg we found a mean value of $139.43 \mathrm{GPa}$ with a standard deviation of $11.82 \mathrm{GPa}$. The remaining material parameters namely the shear moduli, the Poisson's ratios, and the Young's moduli in direction transverse to the fibers were estimated based on former test results with the same materials, specifications of the manufacturer, and by comparison with data of similar material systems, because 
these values do less influence the simulation results for the chosen load case or are more difficult to measure. The material data used for the simulation is shown in table 1.

\begin{tabular}{|c|c|c|c|c|}
\hline$E_{11, \text { Twill }}$ & $62.3 G P a$ & $E_{11, U D}$ & $139.43 G P a$ \\
\hline$E_{22, \text { Twill }}$ & $62.3 G P a$ & $E_{22, U D}$ & $8 G P a$ \\
\hline$E_{33, \text { Twill }}$ & $5.5 G P a$ & $E_{33, U D}$ & $4 G P a$ \\
\cline { 2 - 5 } & $G_{12, \text { Twill }}$ & $5 G P a$ & $G_{12, U D}$ & $5 G P a$ \\
\cline { 2 - 5 } Material properties & $G_{31, \text { Twill }}$ & $5 G P a$ & $G_{31, U D}$ & $5 G P a$ \\
\cline { 2 - 5 } & $G_{23, T w i l l}$ & $4.5 P a$ & $G_{23, U D}$ & $3.1 G P a$ \\
\cline { 2 - 5 } & $\nu_{12, T \text { will }}$ & 0.05 & $\nu_{12, U D}$ & 0.3 \\
\cline { 2 - 5 } & $\nu_{13, \text { Twill }}$ & 0.3 & $\nu_{13, U D}$ & 0.3 \\
\hline$\nu_{23, \text { Twill }}$ & 0.3 & $\nu_{23, U D}$ & 0.3 \\
\hline
\end{tabular}

Table 1: Material properties

\subsection{Experimental set-up}

Figure 5 shows the experimental set-up. Tensile tests were carried out in a tensile machine Zwick Model 1474 with a load cell of $100 \mathrm{kN}$. The samples were clamped on both ends. To equally distribute the introduced load to the samples, we glued two steel plates on both ends. The load was introduced at the maximum amplitude of the sample where we do not expect any rotation of the unit cell. The tests were displacement-controlled resulting in a global strain of up to $6 \%$ and the reaction force was measured.

To measure the displacement a digital image correlation (DIC) system was used from Correlated Solutions. The DIC configuration consisted of 
the cameras Prosilica GT3400 with a resolution of 3384 x 2704 px and a maximum frame rate of $12.7 \mathrm{fps}$. In a post-processing step the principal strain on the surface of the samples was calculated using the DIC-software VIC-3D of Correlated Solutions.

Figure 5: Experimental set-up: Sample mounted on the testing machine

\subsection{Experimental results and validation}

In this section the experimental results are presented and compared to the numerical results. For the simulation we used the same geometry and material properties as described before. An uncertainty was the thickness where values between $1.8 \mathrm{~mm}$ and $2.1 \mathrm{~mm}$ were measured. Therefore, we use a range of numerical results between these thickness values.

In a post-processing routine the results for the entire samples were reduced and averaged to the results for one unit-cell. To identify a single unit cell we used a MATLAB code to identify the peaks in the geometry (maximum amplitude). The force measurements were transformed to line loads by dividing it by the sample width.

Figure 6 shows the axial displacement of sample 5 of the first lay-up during the experiment. The displacement has a homogeneous distribution in $\mathrm{x}$ direction along the width of the sample with no visible edge effects. Hence the displacements do not depend on the x direction. All the unit-cells behave very similar which means that we could reproduce the unit-cell approach. 
Figure 6: Axial displacement for sample 5 of lay-up configuration 1

\subsubsection{Strain-force measurement}

Figures 7 to 9 show the global enforced strain-to-force curves for the three different lay-ups. The enforced global strains are proportional to the enforced displacement calculated with $\epsilon_{\text {enforced }}=\Delta u_{\text {enforced }} / p$. To have a better comparison with the simulation, the settling processes due to the compliance of the test machine in the beginning of the experiment were removed in the displacement-to-force curves. The experimental data is illustrated with dotted lines and the grey areas represent the range of numerical results for different laminate thicknesses.

We can see that the samples behave linear in terms of the force-strain response. The laminate thickness has a very high influence on the numerical simulation, because the mechanical response is mainly influenced by local bending in the corrugated laminate. The thickness influences the local bending by the power of three.

For lay-up 1 we observe a very high reproducibility of the experimental stiffness of the samples. The experiments have a very good agreement with the simulation considering the uncertainties in the thickness measurements. The samples with lay-up 1 show the most compliant behavior.

The samples with lay-up 2 show the stiffest behavior. The experimental results have a good reproducibility and they show a very good agreement with the numerical simulation.

The samples with lay-up 3 have a higher scatter than the other exper- 
iments. The averaged stiffness of the experiments is $3.3 \%$ stiffer than the simulated stiffness for a laminate with a thickness of $2 \mathrm{~mm}$. This is stiffer in comparison with the other two lay-ups where the averaged experimental stiffness was lower than the numerical one for a $2 \mathrm{~mm}$ thick laminate. The experimental and numerical results still show a good agreement.

Figure 7: Global-strain-to-force curve for lay-up 1

Figure 8: Displacement-to-force curve for lay-up 2

Figure 9: Displacement-to-force curve for lay-up 3

\subsubsection{Local strain measurement}

Figure 10 shows the strain measurement for sample 5 of lay-up-configuration 1 at a global axial displacement of $10 \mathrm{~mm}$. The local strain is measured on the surface of the sample in global $y$ direction. We observe negative strain values which correspond to local compression at the highest peaks at $z=h+t / 2$. Positive strain values which correspond to local tension occur at $z=h-t / 2$. The negative strain absolute values are higher than the positive values, because they correspond to a measurement at maximum distance from $z=0$. We can see that all unit-cells behave similar and that the measured values do not change in $x$ direction along the sample width. The measured strains are noisier than the displacements, since it is a secondary measurement result. 
Figure 10: Axial strain for sample 5 of lay-up configuration 1

To compare the experiments with the simulation, we evaluated the local strain values at the maximum amplitude $z=h+t / 2$ for both the experiment and the simulation as shown in figure 11 . The measured strain values were averaged over the width. The simulation of the surface strain was carried out for a global enforced strain of $1 \%$ and $2 \%$ for a sample with a total thickness of 1.9 and $2 \mathrm{~mm}$.

Figure 11: Evaluation of the strain for a) the experiment and b) the simulation

Figures 12 to 14 compare the local strain measurements with the simulated strains with respect to the enforced displacement. For lay-up 1 we observe a very good reproducibility of the experimental result and also a very good match with the numerical simulation. For lay-up 2 the measured strains have a scatter of about $10 \%$. Also the numerical results have a deviation of around $10 \%$. For lay-up 3 the measured strains have a very good reproducibility. In comparison with the numerical simulation, we can observe a relative error of around $7.5 \%$ for the simulated laminate with a thickness of $2 \mathrm{~mm}$ and around $11 \%$ for a thickness of $1.9 \mathrm{~mm}$.

Overall we can see that the simulation for laminates of $2 \mathrm{~mm}$ thickness are closer to the experimental results then the simulation of $1.9 \mathrm{~mm}$. 
Figure 12: Strain measurement for lay-up 1

Figure 13: Strain measurement for lay-up 2

Figure 14: Strain measurement for lay-up 3

\subsubsection{Measurement error}

The calibration error of the DIC test set-up which is the standard deviation of residuals for all views was 0.05 in average. The DIC software indicated this as a low calibration error meaning that a good calibration was achieved. The average projection error for the displacement measurements with the DIC was $0.005 \mathrm{~mm}$. Hence, we assume to have very accurate measurement results for the displacements.

\subsection{Discussion of the experiments}

The experimental results show that the main assumptions of the model are correct. We observe a linear behavior of the samples due to their relative thickness. We could reproduce the unit cell behavior and the generalized plane strain state for both the displacement and strain measurements. The experimental results show a low scatter and have a very good agreement with the numerical results. As expected the samples with lay-up 1 show the most compliant behavior whereas lay-up 2 shows the stiffest behavior. This can be explained considering that the axial deformation of the corrugation is mainly influenced by local bending. In lay-up 2 the stiff UD-layers are placed further 
away from the mid-face line than in lay-up 3, hence the samples with lay-up 2 can better withstand the local bending.

The local strain measurements show negative values where we expect local compression due to the local bending. They have positive values where we expect local tension. The highest strain values are observed at maximum amplitude as expected. The strain measurement shows a good agreement with the numerical results, although it was a secondary measurement and includes a certain noise. These results indicate that the post-processing of our model where we calculate stresses and strains is correct. Hence, we can assume that the numerical calculation of the interlaminar stresses (which are of interest in the present study) represents the reality, although it is not possible to measure them directly.

The laminate thickness has a high influence on the stiffness results, because it influences the local bending by the power of three. The thickness measurements of the samples might include some excess resin which could explain why the simulation is slightly stiffer than the experiment for lay-up 1.

Our FE model assumes that the material properties are homogeneous within one layer according to the classical laminate theory. However, this does not exactly apply for twill layers. A micro-mechanical study [26] showed that the classical laminate theory highly overestimates the bending stiffness for twill layers. However, it also showed that for a three-ply laminate the bending stiffness is almost homogeneous as calculated with the classical laminate theory. This could be a reason why our model predicts stiffness slightly too high than the experiments for the twill lay-up. 
Further discrepancies between the experiments and simulation might be caused by the uncertainties in the material data. First, we had some scattering in the material measurements and secondly we cannot take it as granted that the material properties in a flat laminate are exactly the same as in the corrugated samples which are more difficult to fabricate. Further some material properties are based on experience.

\section{Numerical investigation of interlaminar stresses}

This section presents the numerical results of the interlaminar stress calculations. It shows the parameter study investigating the influence of geometry and laminate lay-up on the interlaminar stress. Further, it sets the interlaminar stress in a relation with the axial compliance of a corrugated laminate. In the discussion we identify favorable geometries and lay-ups in order minimize the interlaminar stresses.

\subsection{Parameter study}

To investigate the influence of geometry and laminate lay-up on the interlaminar stresses, a parameter study was conducted. We investigated the influence of the amplitude to periodic-cell-length ratio $h / p$ and four different layups for both the circular sections and the sinusoidal shape.

The amplitude to periodic-cell-length ratio was varied between 0.05 and 0.75 for the circular sections and 0.05 and 0.45 for the sinusoidal shape in steps of 0.025 . The range of geometries that we cover with that investigation is shown in figure 15 .

We studied four different lay-ups. Three of them were the lay-ups already presented in section 3.1 Sample Design and the forth was a lay-up with eight 
Figure 15: Investigated range of amplitudes for a) circular sections and b) sinusoidal shape

UD-layers made from the same material as in lay-up 2 and 3.

As interlaminar stresses, we considered the shear stress $\sigma_{s t}$ as well as the through-thickness stress $\sigma_{t}$. To be able to compare the different geometries to each other, the interlaminar stresses were normalized with the intralaminar stress $\sigma_{s}$ that points along the midface-shape.

Further, we studied the influence of the different lay-ups and amplitudes on the compliance of the corrugated sheets.

\subsection{Numerical results}

\subsubsection{Interlaminar stress distribution}

Figure 16 to 19 give a qualitative overview over the interlaminar stresses, namely the through-thickness and shear stresses, in the considered geometries. For quantitative comparison between the samples each illustration contains the maximum occurring interlaminar stress value $\sigma_{\max }$. Figure 16 and 17 show the through-thickness stress distribution for both geometries at an amplitude to periodic-cell-length ratio of $h / p=0.25$. We observe the maximum through-thickness stress at the position of maximum amplitude at $z=h$. The stresses are zero at the surface as it is required to fulfill the mechanical equilibrium. The through-thickness stresses are higher for the sinusoidal shape. The values also vary between the different lay-ups. Lay-up 4 with only UD layers show the highest through-thickness stresses. We find the lowest stresses for lay-up 1 with only twill layers.

Figure 18 and 19 illustrate the shear stresses for the circular sections 
Figure 16: Through-thickness stress distribution for the circular sections

Figure 17: Through-thickness stress distribution for the sinusoidal shape

and the sinusoidal shape respectively. The amplitude to periodic-cell-length ratio is $h / p=0.25$. We observe the highest shear stresses for $z=0$. This can be explained considering that the applied tensile force it transferred by a transverse force through the local thickness. The shear stresses are in a similar range for the circular sections and the sinusoidal shape. The shear stresses differ between the investigated lay-ups. We observe the highest shear stresses for lay-up 4 consisting of only UD layers. The lowest value we find for lay-up 1 with only twill layers.

Figure 18: Shear stress distribution for the circular sections

Figure 19: Shear stress distribution for the sinusoidal shape

\subsubsection{Results of the parameter study}

Figure 20 shows the influence of the amplitude to periodic-cell-length ratio $h / p$ and the lay-up on the through-thickness stress to intralaminar stress ratio $\sigma_{t} / \sigma_{s}$. For the sinusoidal shape we observe a high increase in the stress ratios for increasing amplitudes due to the higher curvature. For the circular 
sections we see an increase in the stress ratios with increasing amplitudes until we reach $h / p=0.25$ which corresponds to a semi-circle. If we further increase the amplitude the stress ratios decrease. This behavior can be explained with the corrugation curvature which is inversely proportional to the corrugation radius. In figure 21 we can see that the amplitude has a similar influence on the curvature as on the $\sigma_{t} / \sigma_{s}$ ratios.

Figure 20: Through-thickness stress to in-plane stress ratio, note that the curves for the circular sections for lay-up 1, 2 and 4 are almost the same

Figure 21: Influence of the amplitude to the corrugation radius and curvature for circular sections, the corrugation radius is calculated according to [21]

The influence of the different lay-ups on the stress ratios depend mainly on the location of the maximum intralaminar stress. In lay-up 1 and 4 the maximum intralaminar stress occurs where we observe the highest bending strains. In the figures 20 and 22 we can see that the $\sigma_{\text {inter }} / \sigma_{\text {intra }}$ values are higher for configuration 3. The maximum intralaminar stress in configuration 3 does not occur at the surface where the highest local bending strain appears. Hence if we normalize the interlaminar with the intralaminar stress we normalize with a lower value in comparison to the other configurations which explains the higher $\sigma_{\text {inter }} / \sigma_{\text {intra }}$ values. In configuration 2 the maximum bending stress appears very close to the surface and is therefore comparable to configuration 1 and 4 . If the maximum intralaminar stress 
occurs at the same point for different lay-ups (or separated by one layer), the interlaminar to intralaminar stress ratio has a low dependence from the lay-up.

Figure 22 shows the influence of the amplitude and the lay-up on the shear stress to intralaminar stress ratio $\sigma_{\text {shear }} / \sigma_{s}$. We observe for both geometries decreasing stress ratios for increasing amplitudes. For configuration 1, 2 and 4 , the lay-up does influence the shear stress to intralaminar stress ratio only little, because the maximum intralaminar stresses occur where we observe the maximum bending strains. For configuration 3 higher stress ratios are observable.

Figure 22: Shear stress to in-plane stress ratio

In figure 23 we see the influence of the amplitude and the lay-up on the axial stiffness $A_{22}$. The axial stiffness decreases with increasing amplitude to periodic-cell-length ratios. The axial stiffness is higher for the sinusoidal shape than for the circular sections. Lay-up 4 where we only have UD-layers is the stiffest configuration whereas layup 1 (only twill layers) is the most compliant configuration.

Figure 23: Axial stiffness

\subsection{Discussion of the numerical results}

In a parameter study we investigated the influence of the corrugation amplitude and the lay-up on the normalized interlaminar stress for two ge- 
ometries consisting of circular sections and sinusoidal shapes respectively. We observed that the through-thickness stress is higher in sinusoidal shapes than in circular sections due to the higher curvature at the location of the maximum through-thickness stress. The shear stresses are in a similar range for both geometries.

The interlaminar to intralaminar stress ratio is only little influenced by the lay-up if the maximum bending stresses occur at the same location.

With increasing amplitude, we observed increasing through-thickness to intralaminar stress ratios for sinusoidal shapes. On the other hand, the shear to intralaminar stress ratios decrease with increasing amplitudes. Hence, if we design flexible skins with sinusoidal shapes, we always deal with a design trade-off between minimizing through-thickness or shear to intralaminar stress ratios.

The through-thickness to intralaminar stress ratios increase in circular sections for increasing amplitudes until a semi-circle is reached. A further increase of the amplitude will decrease the through-thickness to intralaminar stress ratios.

According to the manufacturer of the used material the interlaminar shear strength to bending strength ratio is 0.047 for the UD prepreg and 0.07 for the twill prepreg. The interlaminar tensile strength can be assumed to be around $70 \%$ of the interlaminar shear strength. Hence the interlaminar tensile to bending strength ratio is around 0.033 for the UD and 0.049 for the twill. In figure 20 we observe that the circular-section shape never exceeds $\sigma_{t} / \sigma_{s}$-ratios of 0.033 whereas the sinusoidal shape reaches the same ratio already at the low amplitude-to-periodic-cell-length ratios of 0.0125. Here, delamination 
and in-plane failures are estimated to occur at the same external load. For amplitude-to-periodic-cell-length ratios greater than 0.25 , the strength ratio decreases to a minimum value of 0.016 for the circular-section shape. Looking at figure 22 we find maximum values for the $\sigma_{\text {shear }} / \sigma_{s}$-ratios of 0.054 for the circular sections and 0.043 for the sinusoidal shapes. Those values also show the risk of a delamination failure. The values for the $\sigma_{\text {shear }} / \sigma_{s}$-ratios decrease to minimum values of around 0.01 for larger amplitudes for both configurations.

We can identify circular sections with large amplitudes as configurations with both low through-thickness and shear to intralaminar stress ratios and a low risk of delamination. Further these configurations also show the highest axial compliance. Hence, they are ideal candidates for flexible skins. An example of such a configuration is shown in figure 24 .

Figure 24: Optimal configuration to reduce normalized interlaminar stresses

\section{Conclusion}

In this paper we investigated the influence of geometry and laminate layup on the interlaminar stresses under tension. We used a numerical model that uses a unit-cell approach and a generalized plane strain state in order to minimize the computational costs. With the used model we are able to analyze corrugated laminates of any thickness and lay-up. The model was therefore very suitable for full-factorial parameter studies for different laminates. We validated the numerical model with experiments where we mea- 
sured the displacements and strains for three different lay-ups. The different lay-ups consisted of twill and UD prepreg. We found very good agreement between the experiments and the simulation for both the displacements and strains. The laminate thickness is a source of uncertainty. It influences the numerical simulation a lot and it cannot be measured very exactly due to excess resin and variation within the samples respectively. Further, with the experiments we could show that the assumptions of the numerical model are valid, namely the linearity for small global strains, the unit-cell approach and the generalized plane strain state.

Using numerical simulation a parameter study was conducted investigating the influence of the corrugation amplitude and lay-up on the interlaminar to intralaminar stress ratio for two geometries consisting of circular sections and a sinusoidal shape. We found that the through-thickness stresses are in general higher in sinusoidal geometries than in circular sections. The shear stresses are in a similar range for both geometries. The interlaminar stresses are higher for stiffer lay-ups, namely pure UD-layers in comparison to more compliant twill layers.

In the parameter study we normalized the interlaminar stresses with the maximum intralaminar stress in order to better compare the different samples. We found that the normalized through-thickness stress increases with increasing amplitudes for the sinusoidal shape. For the circular sections, we first observed an increase in the through-thickness to intralaminar stress ratio up to an amplitude to periodic-cell-length ratio of 0.25 which corresponds to a semi circle. If we further increase the amplitude the normalized through-thickness stress decreases again. We found out that the normalized 
shear stress decreases for increasing amplitudes for both geometries and all the lay-ups.

Therefore, we conclude that corrugated sheets with high amplitudes are ideal to minimize the interlaminar to intralaminar stress ratio for both the through-thickness and shear stress. If we design structures with sinusoidal shape on the other hand, there is always a trade-off between minimizing normalized through-thickness or shear stresses. Corrugated laminates consisting of circular sections with high amplitudes are ideal candidates for flexible skins since they have an axial compliance that is up to three orders of magnitude higher than corrugations with very small amplitudes. In further studies we could investigate the failure behavior, in particular delamination, caused by interlaminar stresses. We could also consider implementing these corrugated laminates in morphing wings.

\section{Acknowledgment}

The authors gratefully acknowledge the support of the Swiss National Science Foundation (Project no. 149200 and grant no. 206021_150729)

\section{References}

[1] F. Previtali, a. F. Arrieta, and P. Ermanni. Double-walled corrugated structure for bending-stiff anisotropic morphing skins. Journal of Intelligent Material Systems and Structures, October 2014.

[2] T. Yokozeki, S. Takeda, T. Ogasawara, and T. Ishikawa. Mechanical properties of corrugated composites for candidate materials of flexible 
wing structures. Composites Part A: Applied Science and Manufacturing, 37(10):1578-1586, October 2006.

[3] C Thill, J Etches, I Bond, K Potter, and P Weaver. Morphing skins. Aeronautical Journal, 112(3216):117-139, 2008.

[4] S. Barbarino, O. Bilgen, R. M. Ajaj, M. I. Friswell, and D. J. Inman. A Review of Morphing Aircraft. Journal of Intelligent Material Systems and Structures, 22(9):823-877, 2011.

[5] C Thill, J D Downsborough, S J Lai, I P Bond, and D P Jones. Aerodynamic study of corrugated skins for morphing wing applications. The Aeronautical Journal, (3407):237-244, 2010.

[6] I. Dayyani, a.D. Shaw, E.I. Saavedra Flores, and M.I. Friswell. The Mechanics of Composite Corrugated Structures: A Review with Applications in Morphing Aircraft. Composite Structures, 133:358-380, 2015.

[7] Y. Xia, O. Bilgen, and M. I. Friswell. The effect of corrugated skins on aerodynamic performance. Journal of Intelligent Material Systems and Structures, 25(7):786-794, February 2014.

[8] M. R. Wisnom and M.I. Jones. Delamination due to interaction between curvature induced interlaminar tension and stresses at terminating plies. Composite Structures, 32(1-4):615-620, 1995.

[9] I. Dayyani, M. I. Friswell, S. Ziaei-Rad, and E. I. Saavedra Flores. Equivalent models of composite corrugated cores with elastomeric coatings for morphing structures. Composite Structures, 104:281-292, October 2013. 
[10] Y. Xia, M. I. Friswell, and E. I Saavedra Flores. Equivalent models of corrugated panels. International Journal of Solids and Structures, 49(13):1453-1462, June 2012.

[11] Y. Xia and M. I. Friswell. Equivalent Models of Corrugated Laminates for Morphing Skins. Active and Passive Smart Structures and Integrated Systems, 7977:79771I-79771I-10, March 2011.

[12] M. Winkler and G. Kress. Deformation limits for corrugated cross-ply laminates. Composite Structures, 92(6):1458-1468, May 2010.

[13] H. Mohammadi, S. Ziaei-Rad, and I. Dayyani. An equivalent model for trapezoidal corrugated cores based on homogenization method. Composite Structures, 131:160-170, 2015.

[14] L. X. Peng, K. M. Liew, and S. Kitipornchai. Analysis of stiffened corrugated plates based on the FSDT via the mesh-free method. International Journal of Mechanical Sciences, 49(3):364-378, 2007.

[15] K.M. Liew, L.X. Peng, and S. Kitipornchai. Nonlinear analysis of corrugated plates using a FSDT and a meshfree method. Computer Methods in Applied Mechanics and Engineering, 196(21-24):2358-2376, April 2007.

[16] R. Roos, G. Kress, M. Barbezat, and P. Ermanni. Enhanced model for interlaminar normal stress in singly curved laminates. Composite Structures, 80(3):327-333, October 2007.

[17] G. Kress, M. Siau, and P. Ermanni. Iterative solution methods for 
damage progression analysis. Composite Structures, 69(1):21-33, June 2005.

[18] F. Fraternali and G. Bilotti. Nonlinear elastic stress analysis in curved composite beams. Computers \& Structures, 62(5):837-859, 1997.

[19] R. a. Shenoi and W. Wang. Through-thickness stresses in curved composite laminates and sandwich beams. Composites Science and Technology, 61(11):1501-1512, 2001.

[20] J. M. Gonzalez-Cantero, E. Graciani, F. Paris, B. Lopez-Romano, and D. Meizoso-Latova. Semi-Analytic Solution of Non-Regularized Unfolding Stresses in Composite Beams Employing a Series Approximation Based on Legendre Polynomials. In International Conference on Composite Materials ICCM20, number July, pages 19-24, Copenhagen, 2015.

[21] G. Kress and M. Winkler. Corrugated laminate homogenization model. Composite Structures, 92(3):795-810, February 2010.

[22] G. Kress and M. Winkler. Corrugated laminate analysis: A generalized plane-strain problem. Composite Structures, 93(5):1493-1504, April 2011.

[23] M. Winkler and G. Kress. Modeling of corrugated laminates. Composite Structures, 109:86-92, March 2014.

[24] J. Barlow. Optimal Stress Locations in Finite Element Models. International Journal for Numerical Methods in Engineering, 10(December 1974):243-251, 1976. 
[25] C. Thurnherr, G. Kress, and P. Ermanni. Interlaminar Stresses in Corrugated Structures made from Anisotropic Materials. In 20th International Conference on Composite Materials ICCM20, number 4418-1, Copenhagen, Denmark, 2015.

[26] O. Soykasap. Micromechanical Models for Bending Behavior of Woven Composites. Journal of Spacecraft and Rockets, 43(5):1093-1100, 2006. 
Figure01

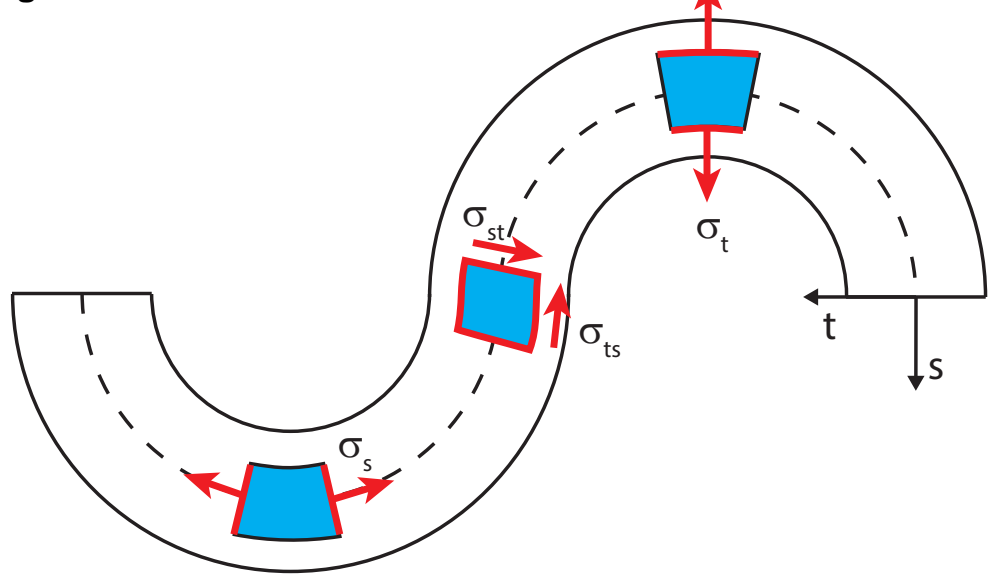




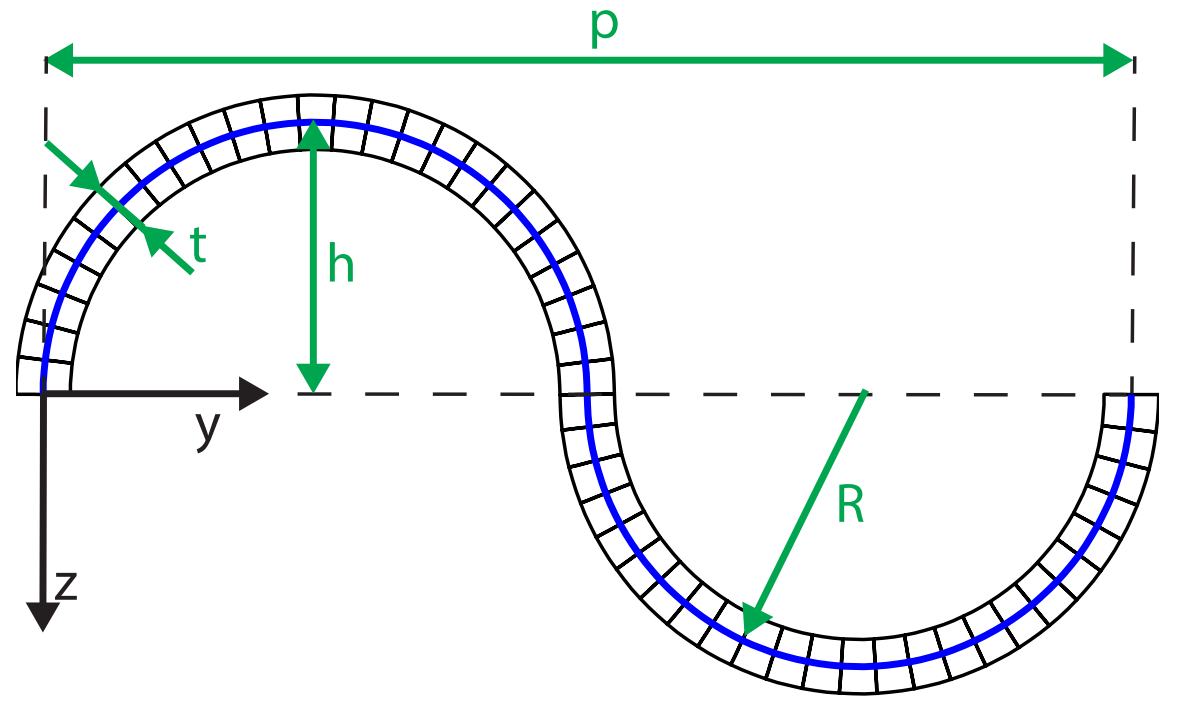

a) Circular sections

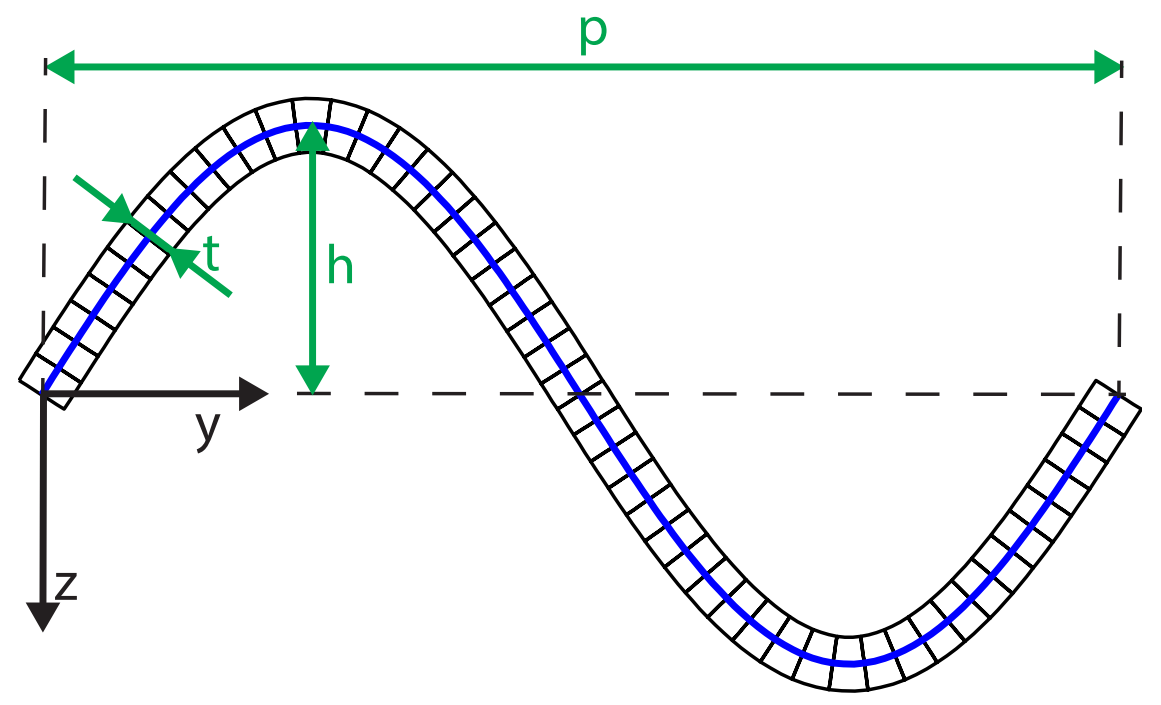

b) Sinusoidal shape 

Lay-up 1

Twill

Twill

Twill

Twill

Twill

Twill

Twill

Twill
Lay-up 2

Twill

UD

UD

Twill

Twill

UD

UD

Twill
Lay-up 3

Twill

Twil

UD

UD

UD

UD

Twill

Twill 
Click here to download high resolution image

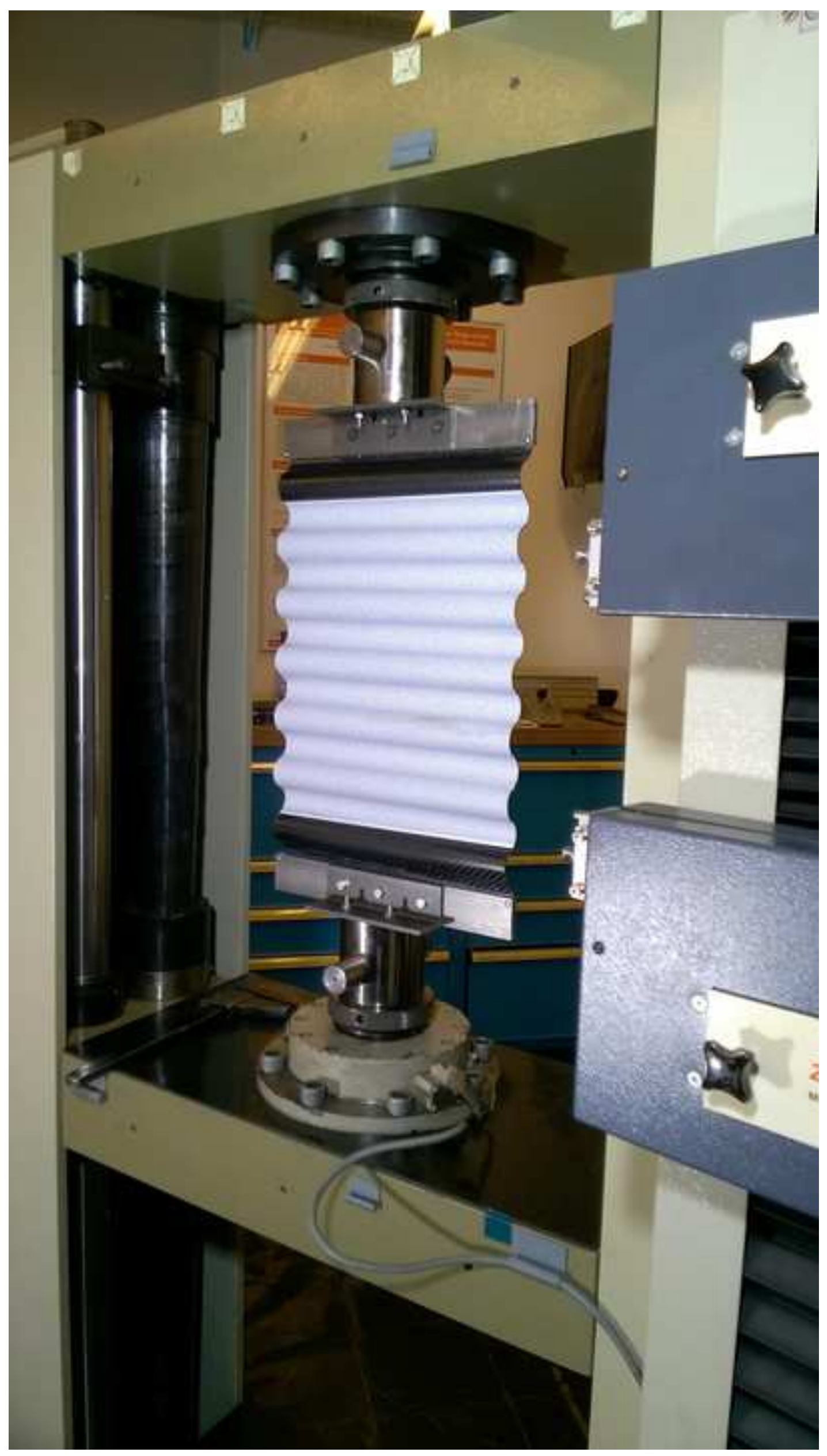




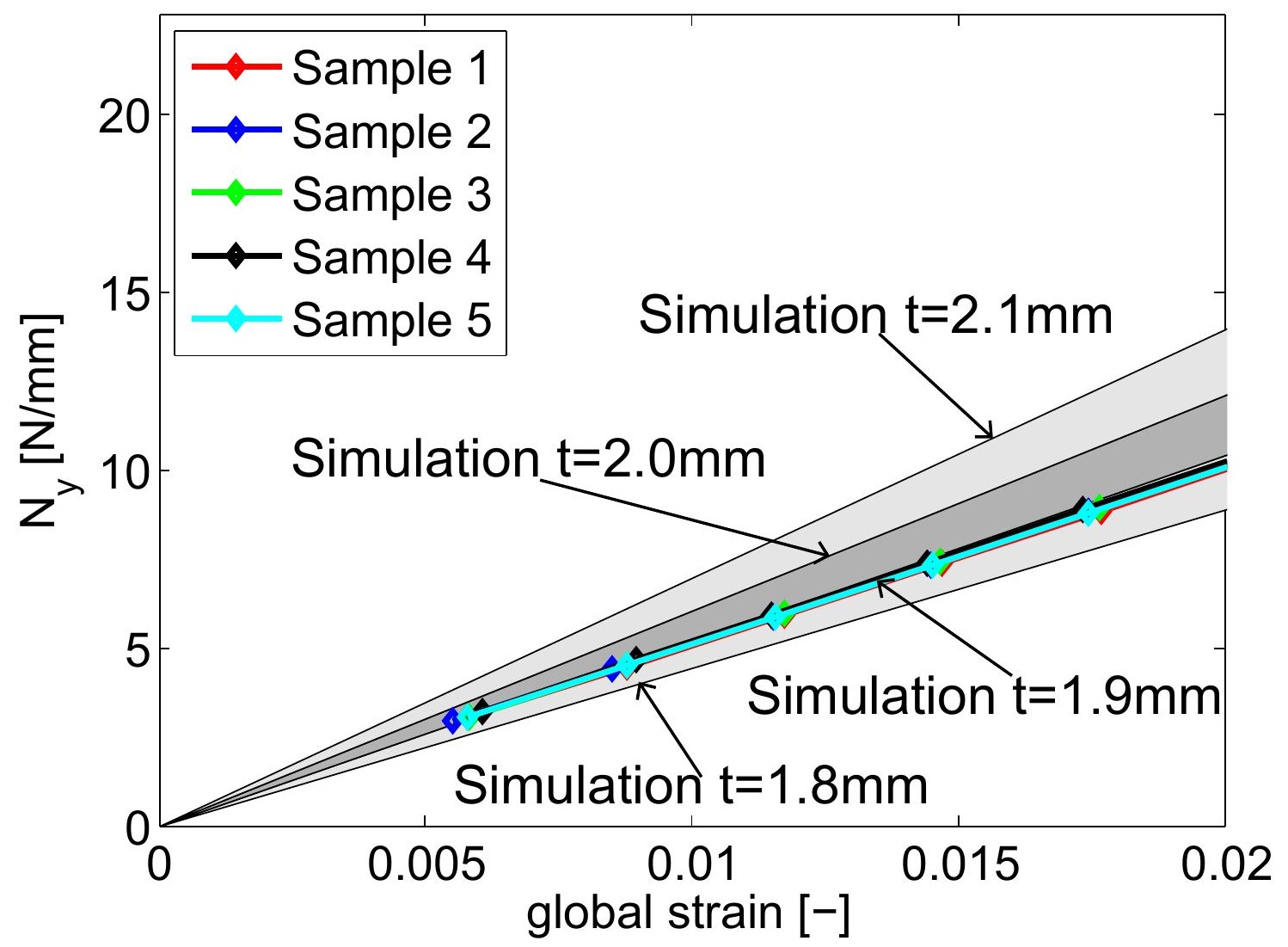




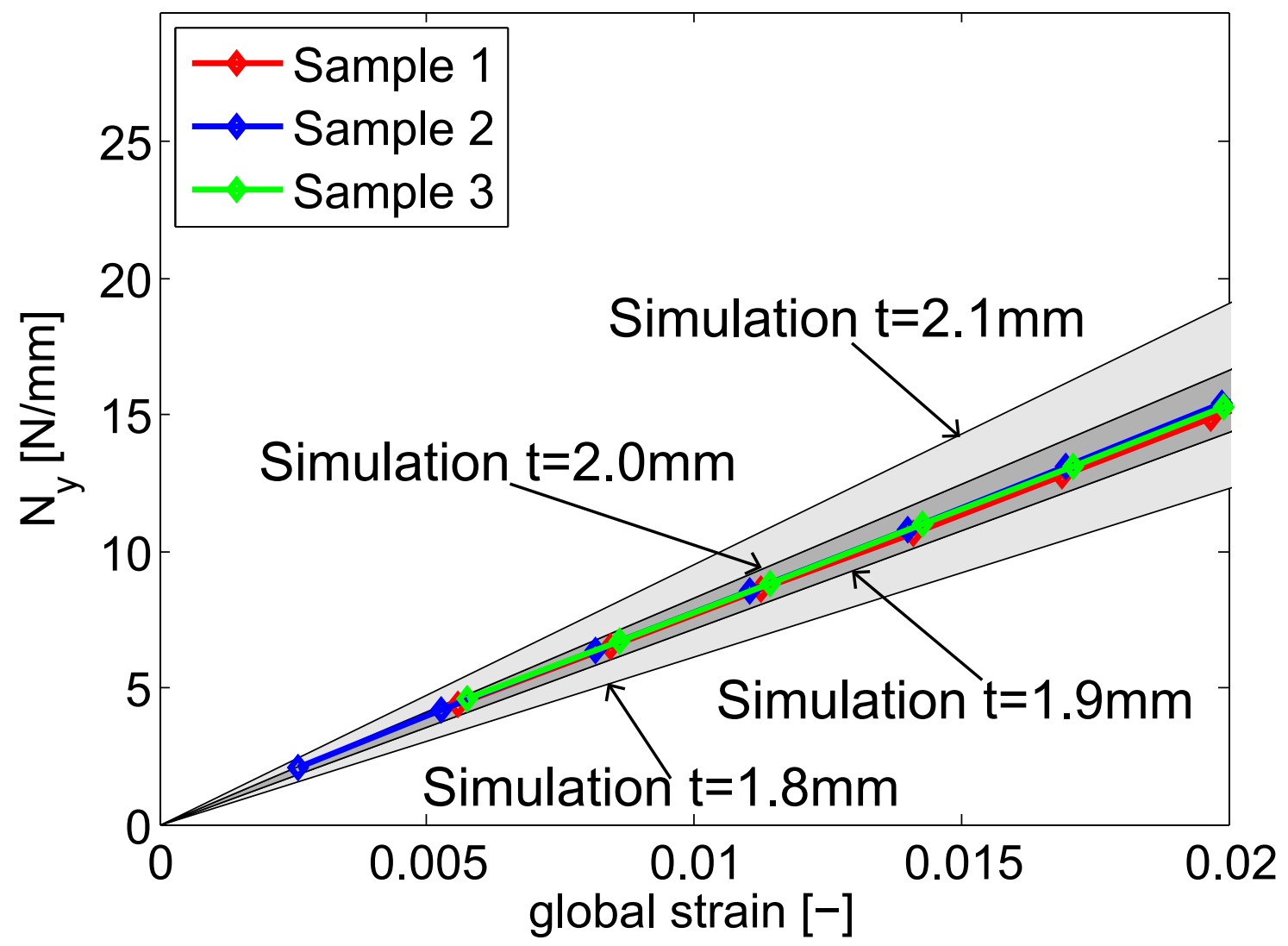




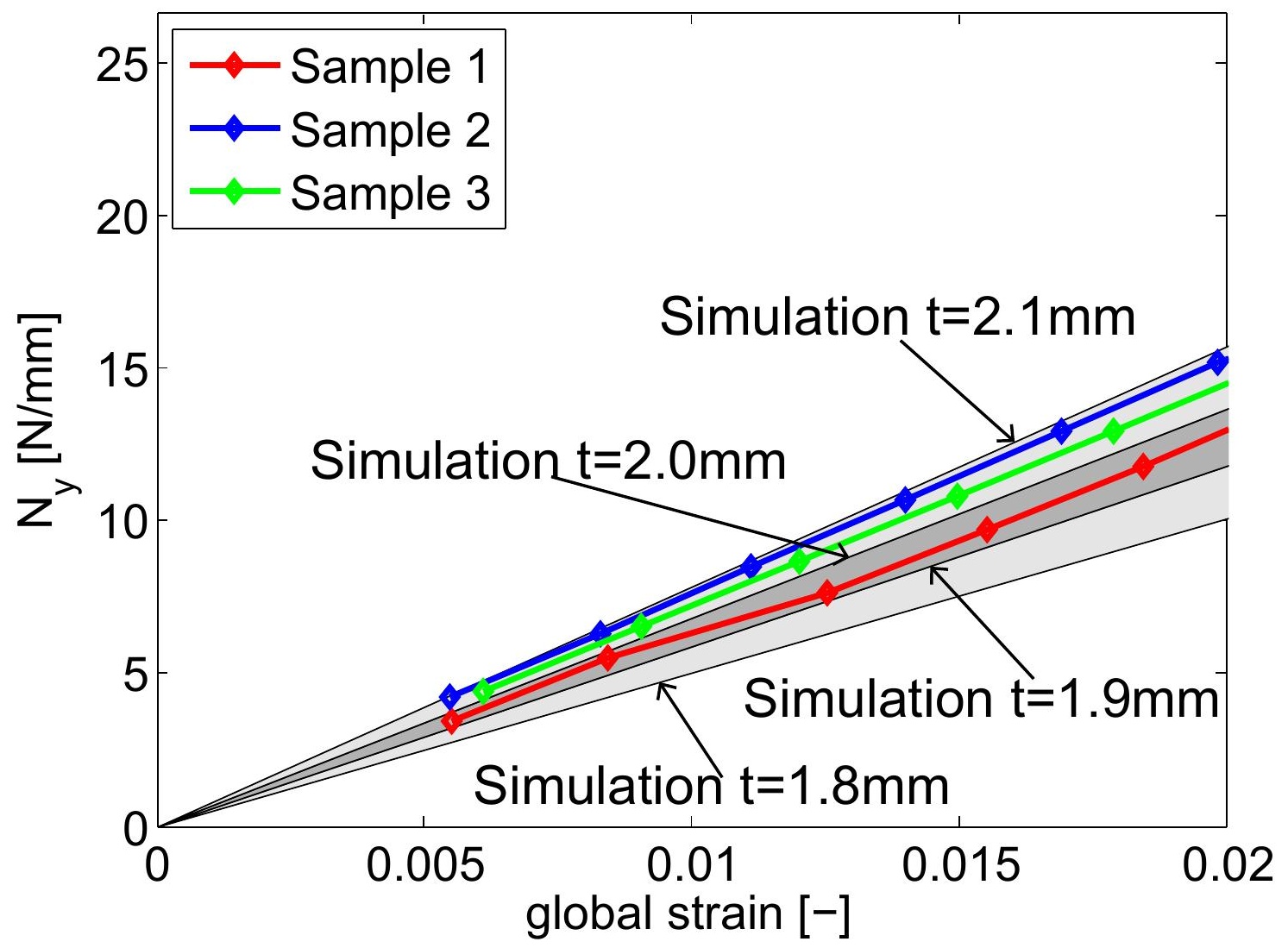


Click here to download high resolution image

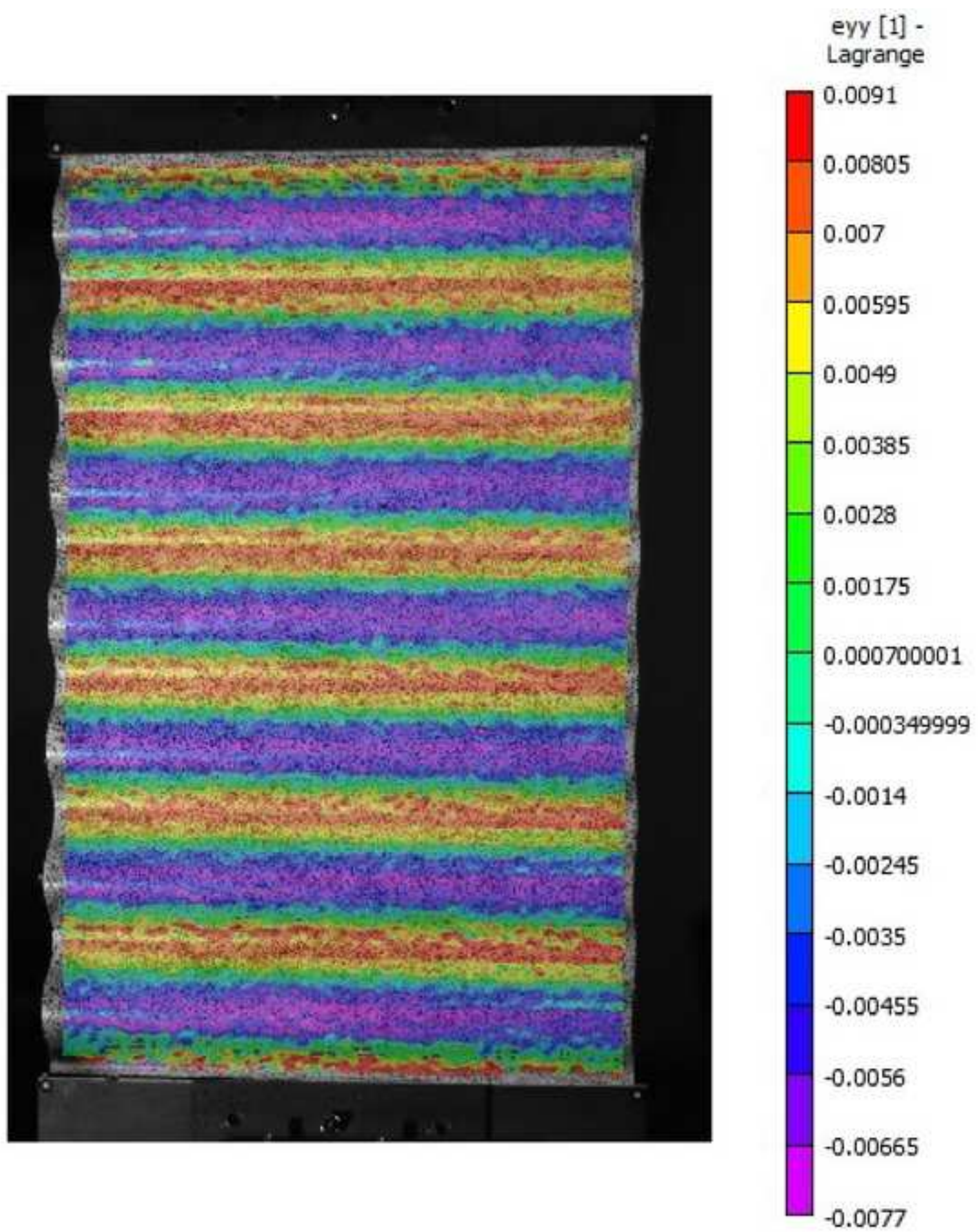


Figure13

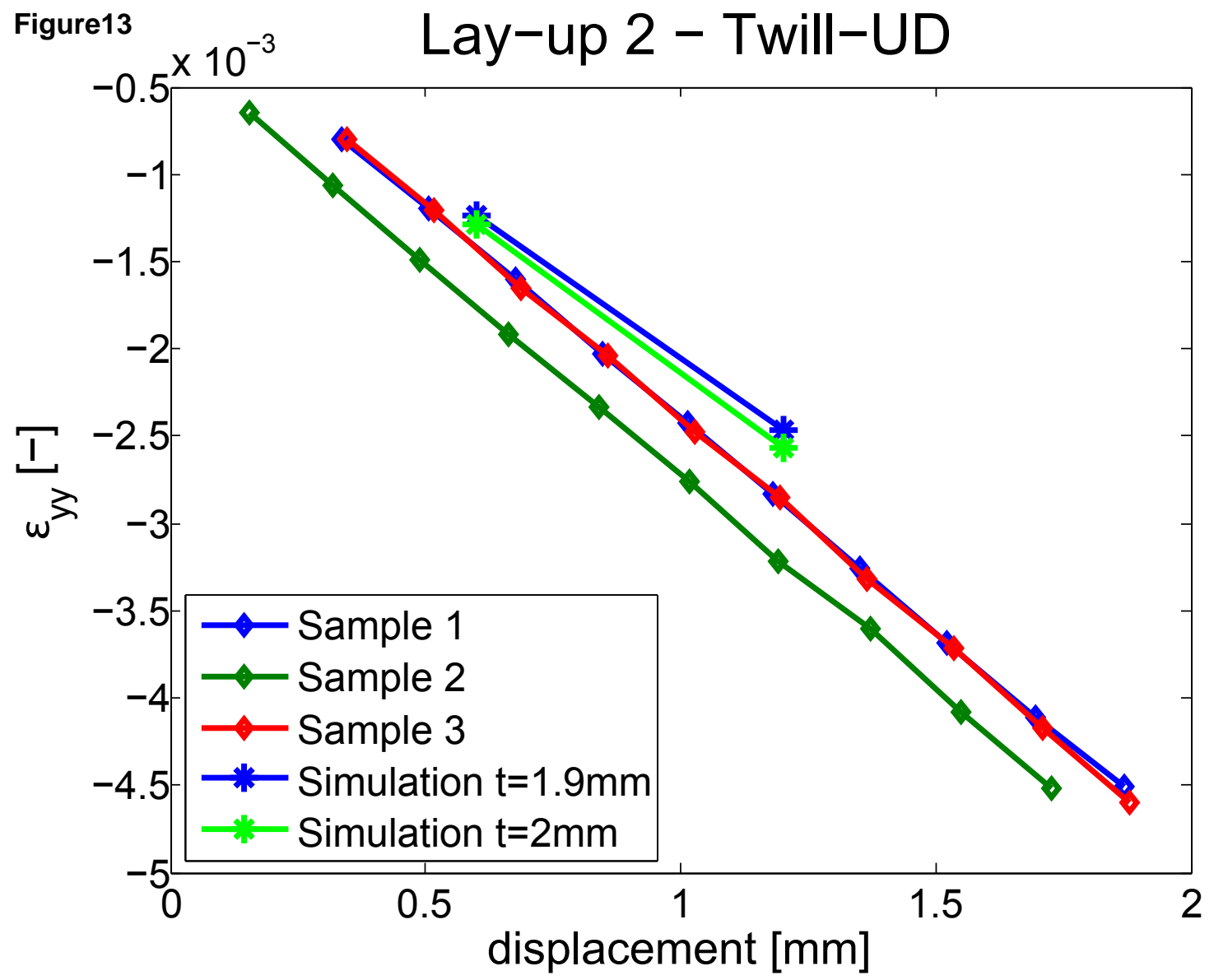


Figure16 Through-thickness stress - Circular sections

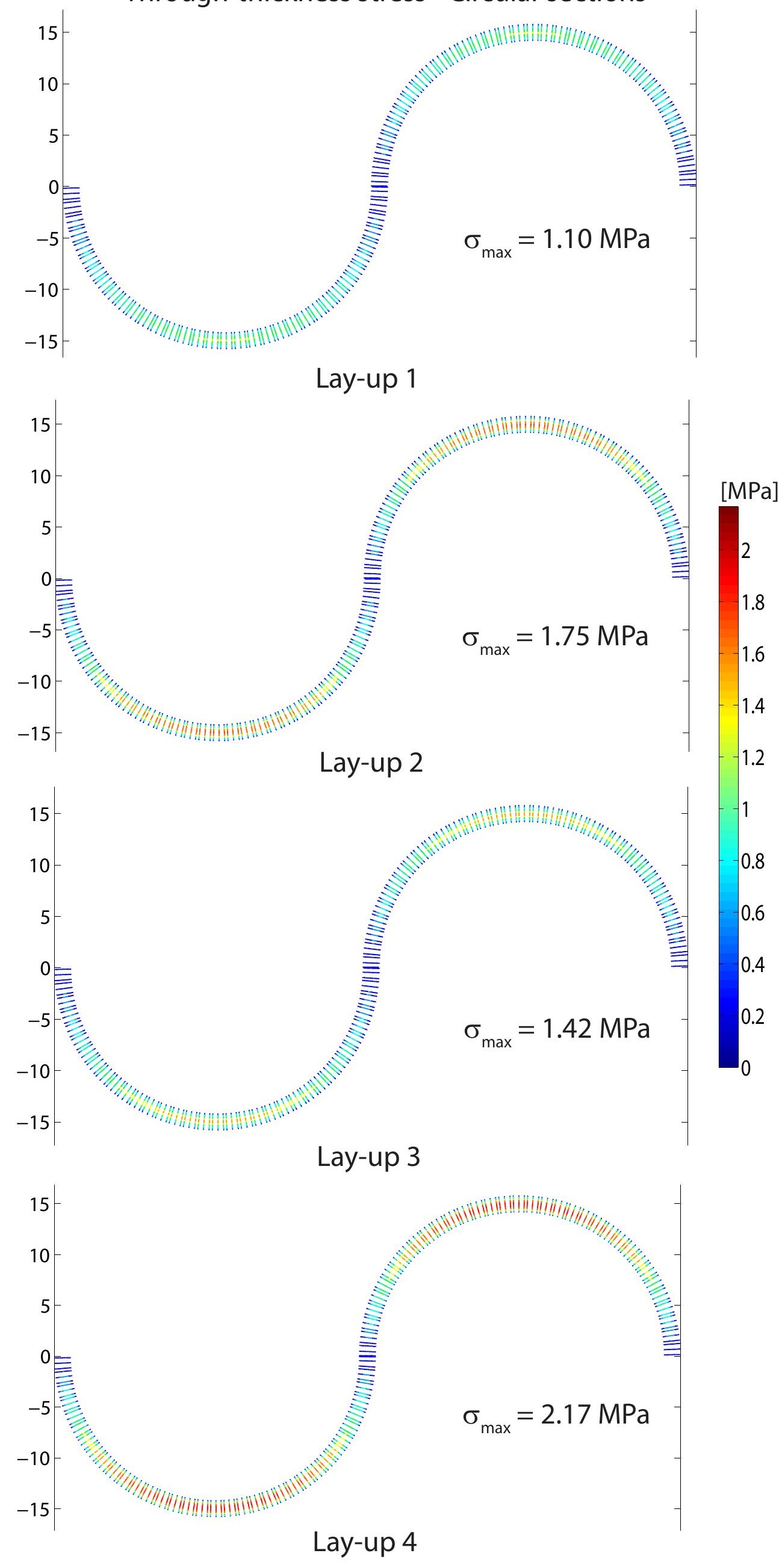


Figure17 Through-thickness stress - Sinusoidal shape

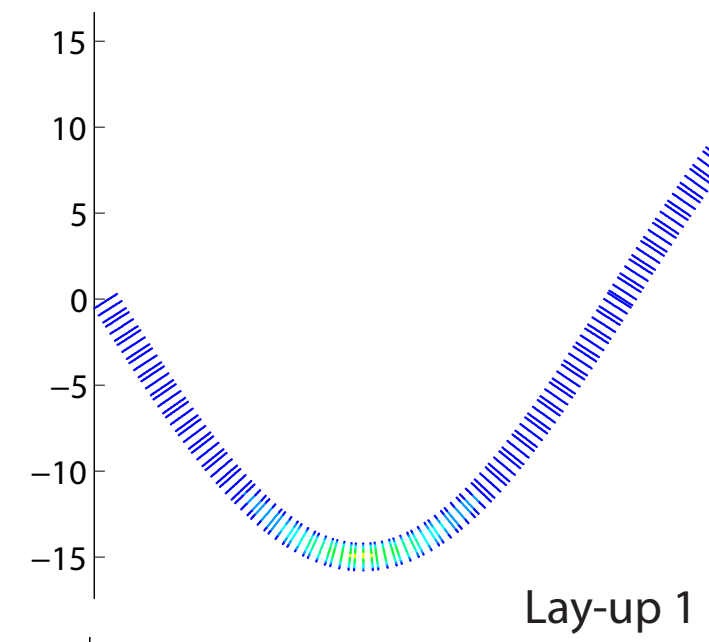

15
10

$$
\sigma_{\text {max }}=3.34 \mathrm{MPa}
$$

Lay-up 1

Lay-up 2

$$
\sigma_{\max }=5.24 \mathrm{MPa}
$$

[MPa]
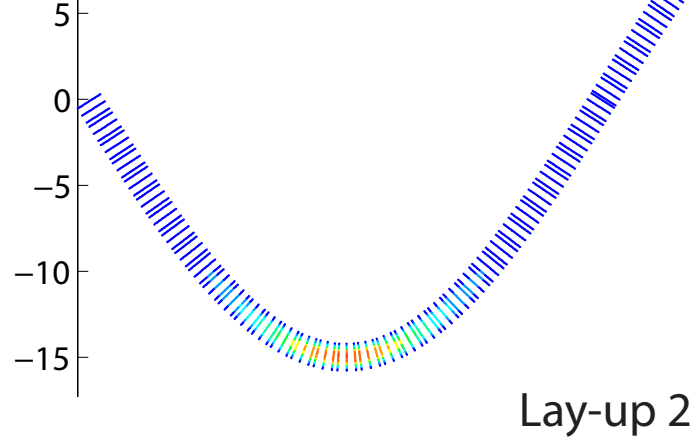

$$
\begin{array}{r}
15 \\
10 \\
5 \\
0 \\
-5 \\
-10 \\
-15
\end{array}
$$

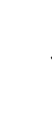

Lay-up 3

$$
\sigma_{\text {max }}=4.21 \mathrm{MPa}
$$

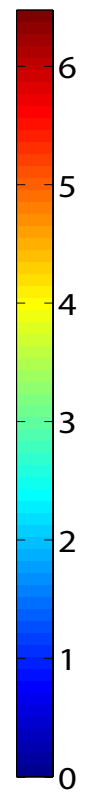

$$
\begin{array}{r}
10 \\
5 \\
0 \\
-5 \\
-10 \\
-15
\end{array}
$$

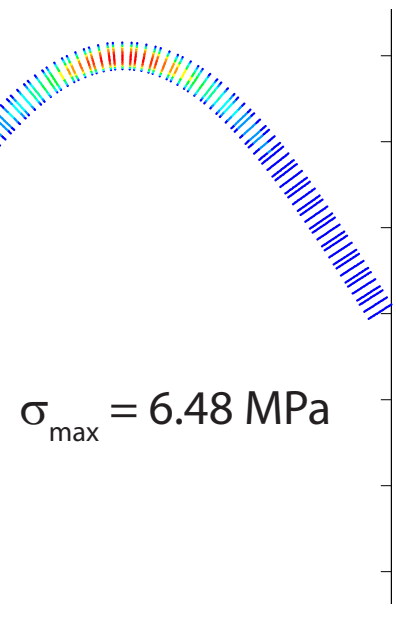




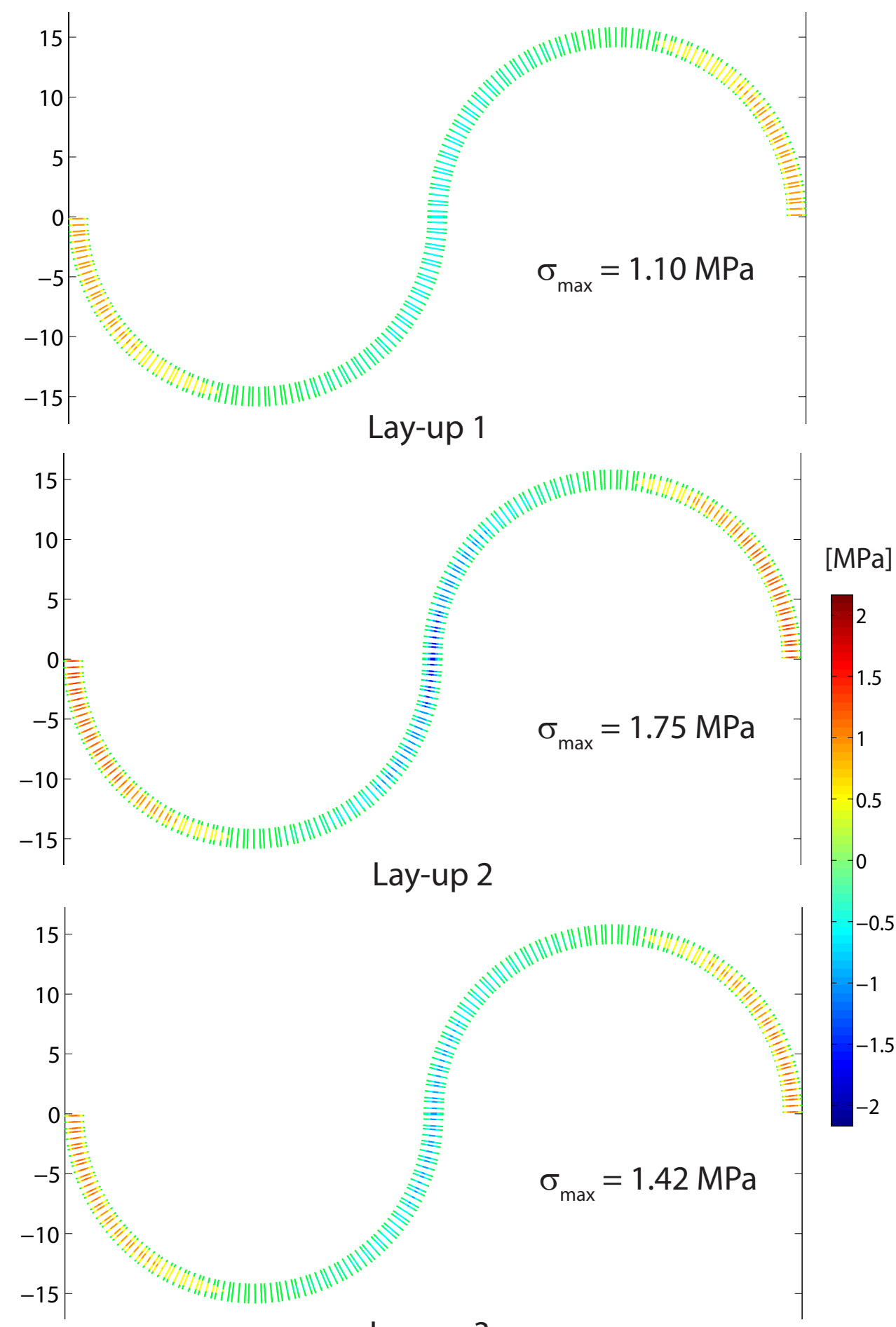

\section{Lay-up 3}
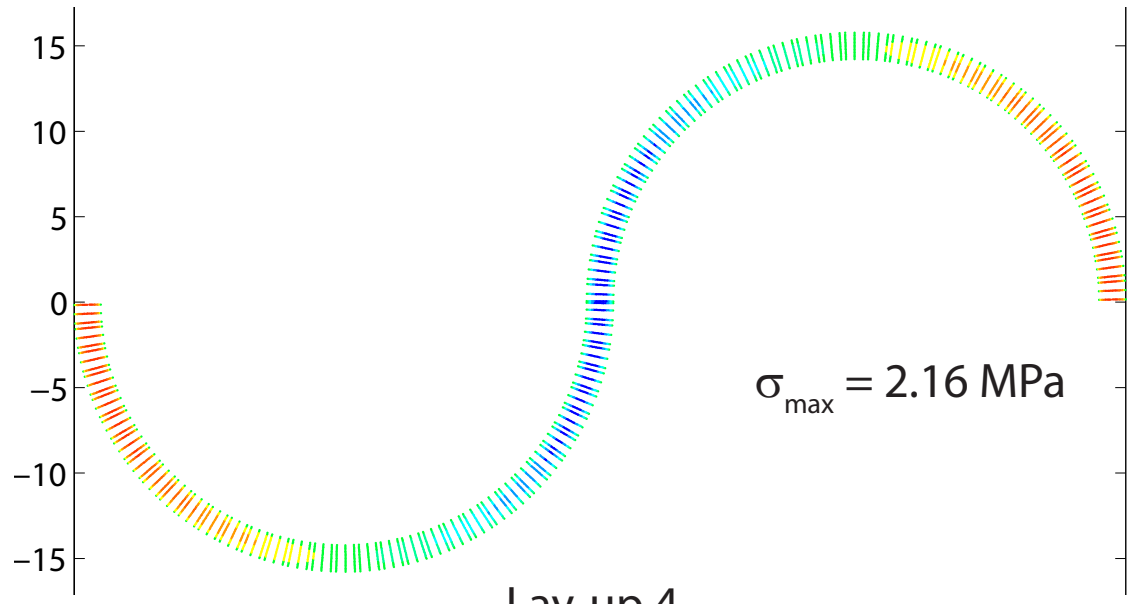


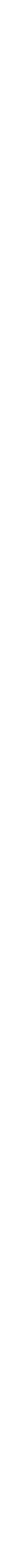




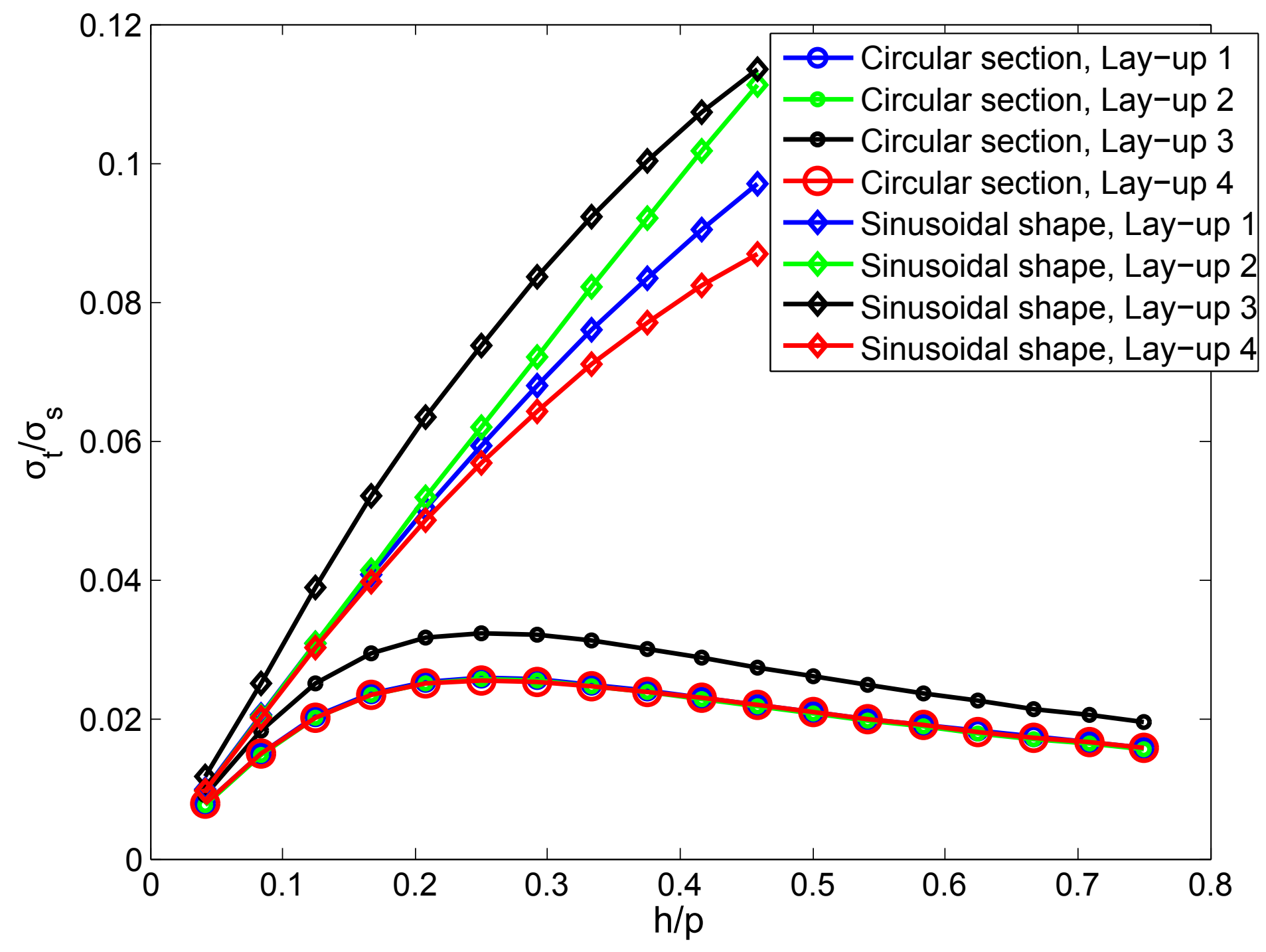




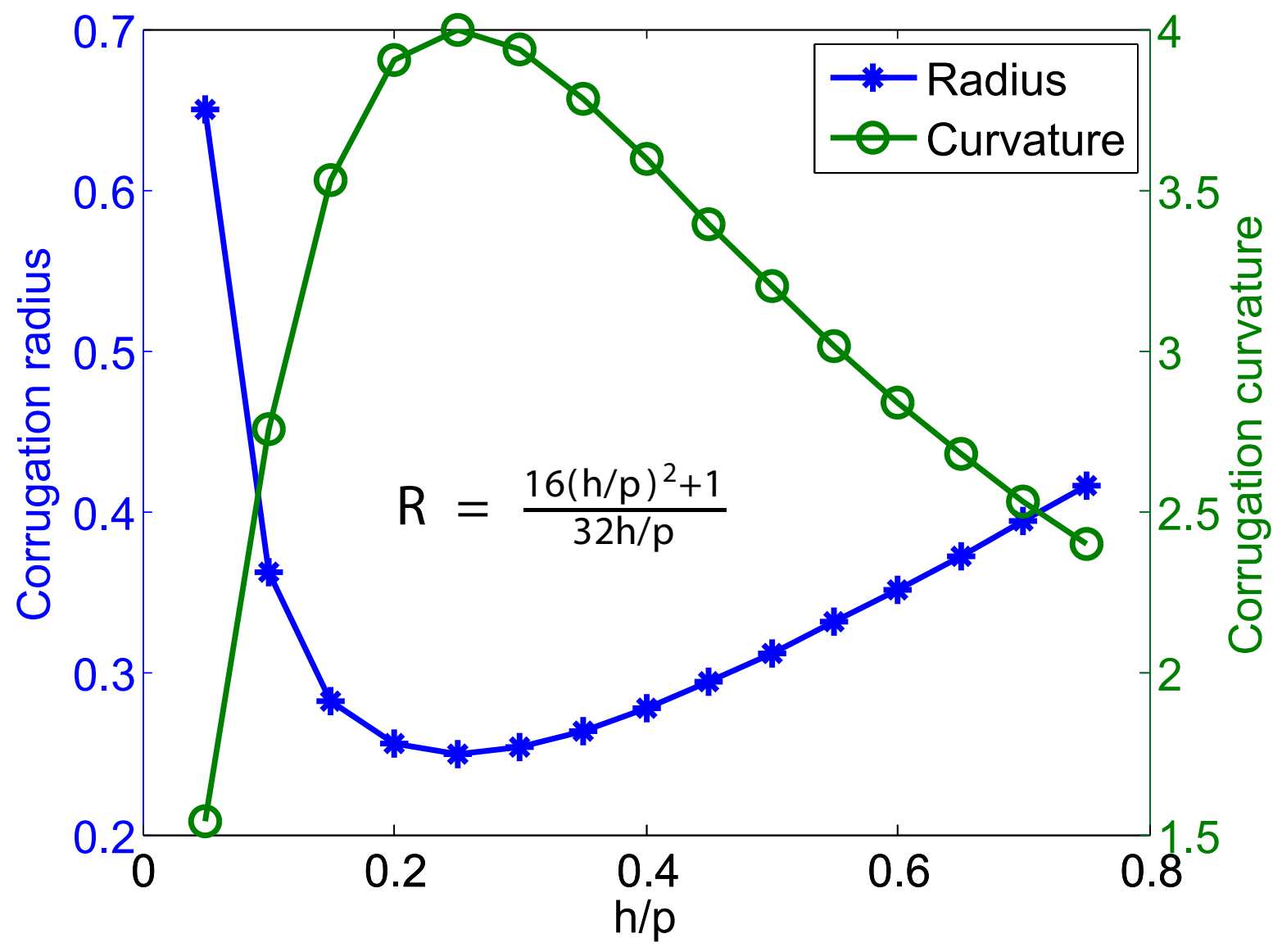




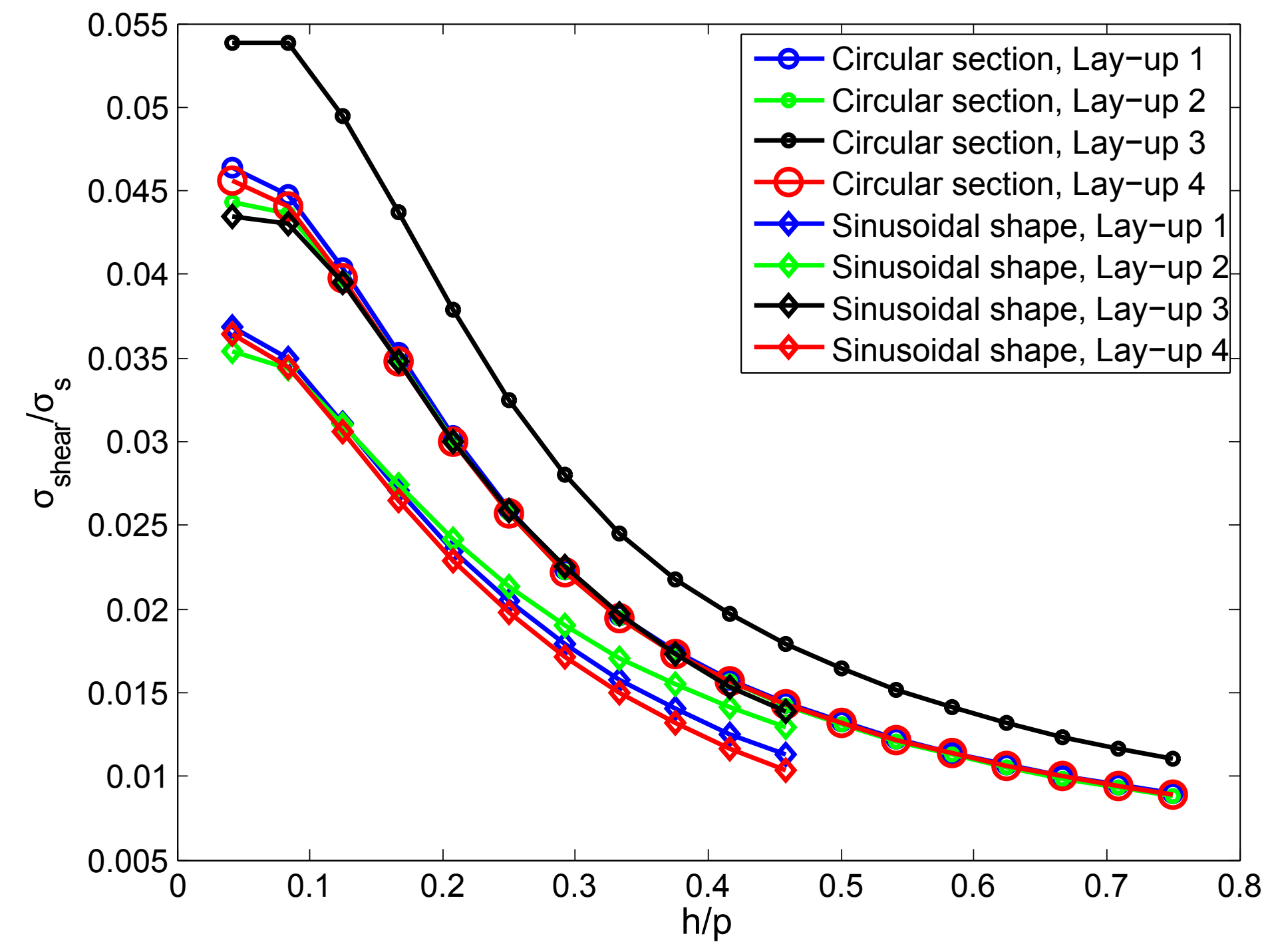




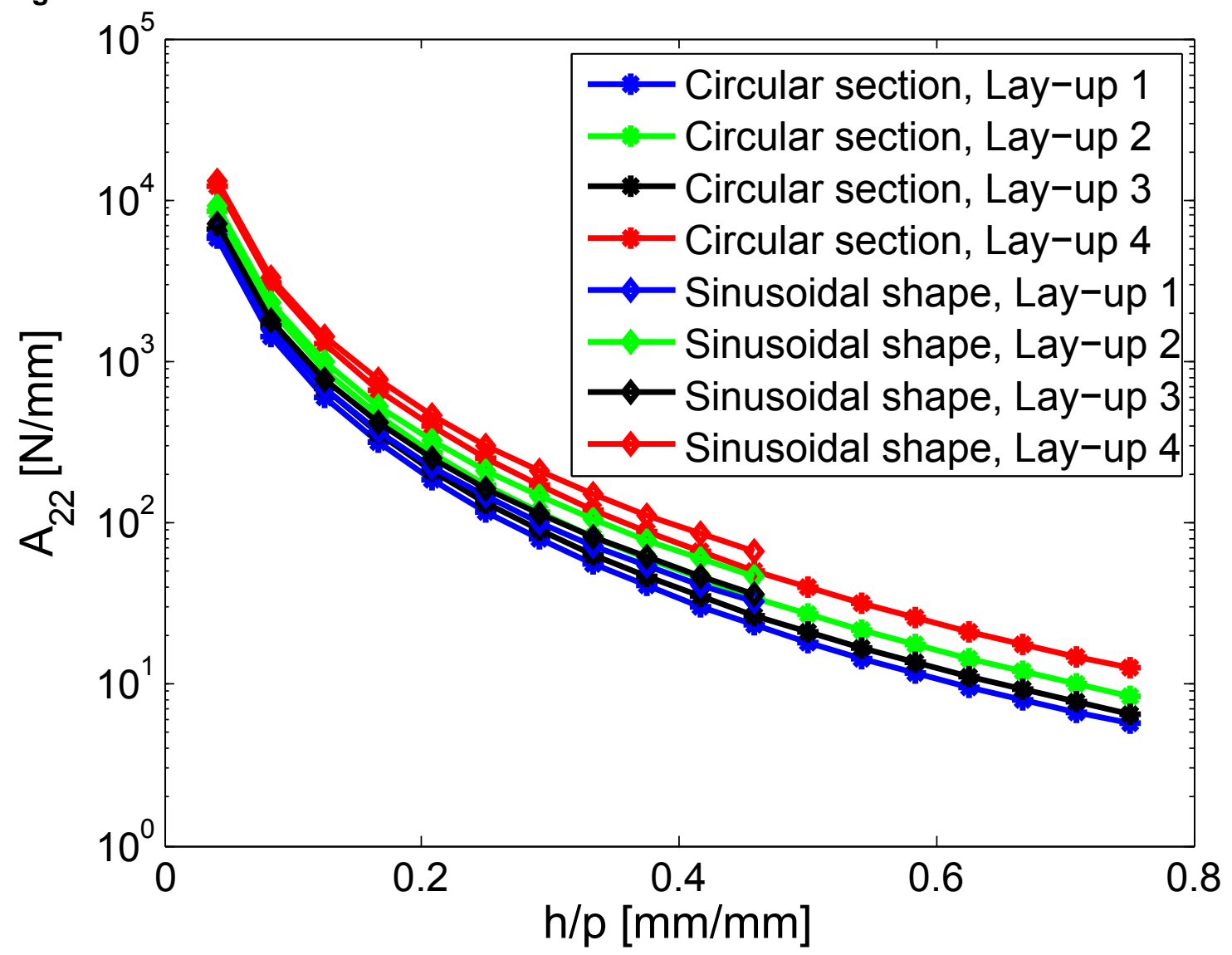

Figure23 


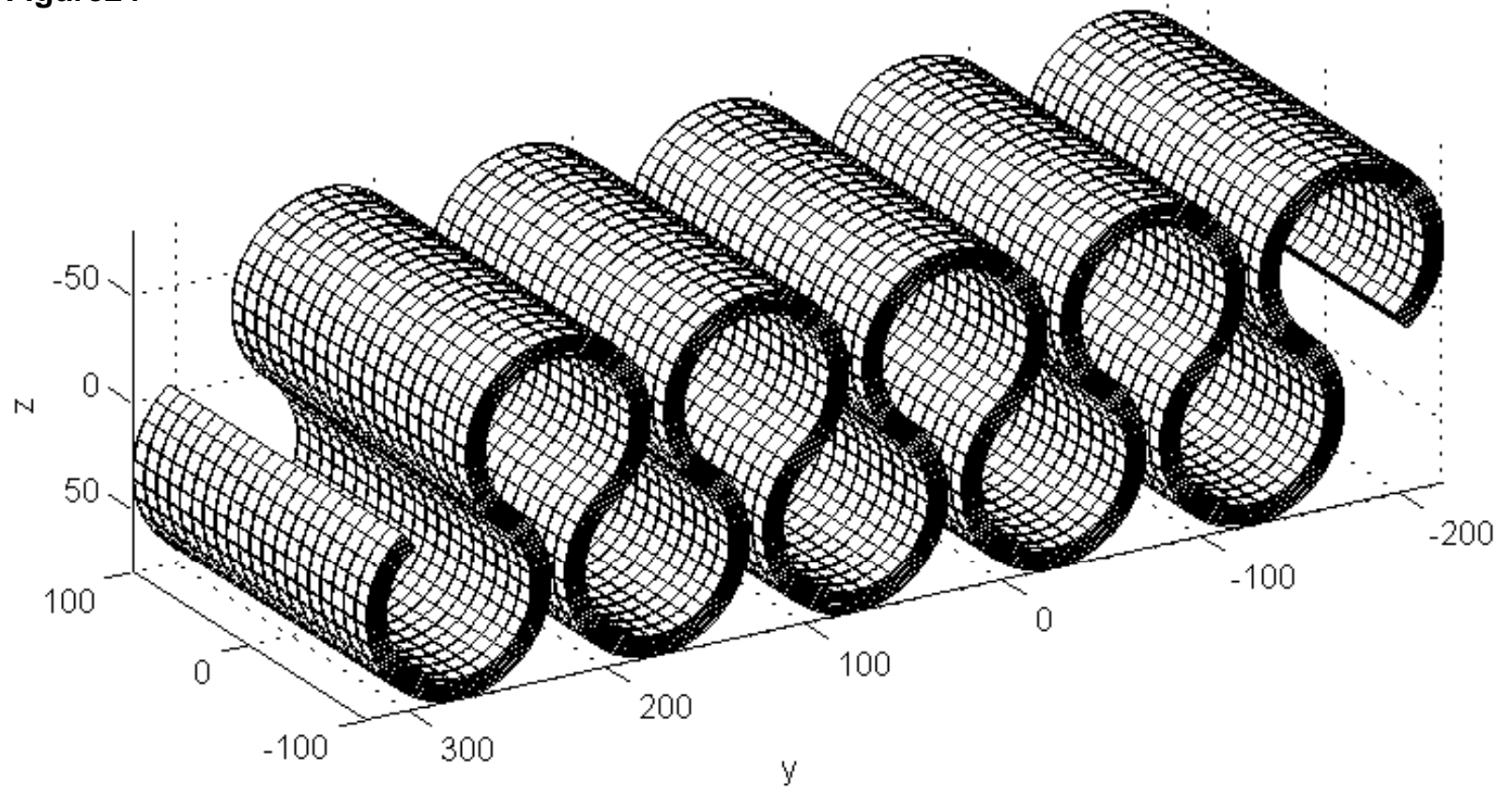

